\title{
Channel adaptive dwell timing for handover decision in VLC-WiFi heterogeneous networks
}

\author{
Xu Bao ${ }^{{ }^{*}+}$, Andrews A. Okine ${ }^{1 \dagger}$, Winfred Adjardjah', Wence Zhang ${ }^{1}$ and Jinsheng Dai ${ }^{2}$
}

\begin{abstract}
The coexistence of visible light communication (VLC) and WiFi elevates indoor wireless communications beyond 4G, by utilizing VLC for high-rate transmission and ensuring reliable mobile connectivity through WiFi. Nevertheless, the high susceptibility of VLC-line-of-sight (VLC-LoS) channel to blocking can result in excessive number of handovers between VLC and WiFi networks leading to huge signaling cost and degradation in overall system performance. Therefore, an efficient inter-system handover scheme is required for a successful integration of the two access technologies. To this end, a channel adaptive dwell vertical handover (CAD-VHO) scheme is presented, in this article, as an improvement on static D-VHO scheme. The width of the dwell timer employed in our scheme is altered based on the rate of blocking and recovery of VLC-LoS channel. As a result, the proposed CAD-VHO scheme is adaptive to the rate and extent of obstruction of VLC-LOS link due to user mobility and surrounding objects. Simulation scenario was set up as a dynamic network environment in an indoor setting with restless and stationary mobile terminals and surrounding mobile object. Based on the simulation results obtained, the CAD-VHO scheme can reduce the number of $\mathrm{VHO}$ s by as much as $80 \%$ whiles achieving a higher average data rate in most cases. Consequently, the proposed CAD-VHO scheme is highly efficient in minimizing VHO signaling cost and enabling a superior broadband service.
\end{abstract}

Keywords: Heterogeneous network (HetNet), Vertical handover (VHO), Line-of-sight (LoS), Dwell timer, Visible light communication (VLC)

\section{Introduction}

Over the last two decades, there has been rapid growth in mobile data traffic due to the surge in the number of multimedia-enabled mobile devices capable of accessing the Internet. Recent studies have found that most of the data consumption occurs indoors [1]. The main challenge for current and future wireless networks is to meet the ever growing capacity demands of multimedia applications such as HD video streaming [2]. Combining an access network having a smaller but denser coverage and another having a wider but less dense coverage can be an effective solution to the traffic demand and network capacity discrepancy $[3,4]$.

Against this backdrop, visible light communications (VLC)-WiFi heterogeneous network (HetNet) has emerged as a promising solution to the capacity and

*Correspondence: xbao@ujs.edu.cn

${ }^{+}$Xu Bao and Andrews A. Okine contributed equally to this work.

'School of Computer Science and Communication Engineering, Jiangsu University, 301 Xuefu Road, 212013 Zhenjiang, People's Republic of China

Full list of author information is available at the end of the article mobility requirements of indoor wireless communications for alleviating the data traffic. Despite providing relatively wide mobile connectivity, WiFi has limited capacity to meet the growing demand for gigabit per second (Gbps) transmissions in the era of multimedia communication. On the contrary, VLC can solve the issue of network capacity since it makes use of the abundant and unregulated light spectrum for wireless communications $[5,6]$. However, the limitation in the coverage of VLC is a drawback. By combining VLC and WiFi, we can have a hybrid communication network that offers high wireless capacity and dependable mobile connectivity [7]. To achieve this feat, the critical issue of vertical handover (VHO) from one network to the other has to be addressed.

In heterogeneous VLC-WiFi networks, VHO is usually necessitated when mobile device moves into or out of the coverage of VLC. Since VLC is a line-of-sight (LoS) technology, its availability to a mobile device cannot always be guaranteed. Besides, the random movement of surrounding objects makes the VLC-LoS link even more vulnerable. To ensure continuity in communication when VLC link is 
lost, VHO to WiFi may be performed [8]. Traditionally, there are two main schemes used for handover decision when VLC channel is not available. In the immediate vertical handover (I-VHO) scheme, the link is switched to WiFi as soon as LoS optical link is lost and switched back when link is recovered. This may lead to ping-pong effects and unnecessary signaling cost if the optical link is blocked for a very short period. For the dwell vertical handover (D-VHO) scheme, VHO controller waits for a fixed duration in anticipation of link recovery. In this scheme, VHO is only performed when VLC channel is still blocked after the expiration of the waiting time [9]. Notwithstanding, the efficiency of D-VHO scheme in a dynamic network environment can be limited by the static nature of its timer.

Some works have been found in literature where the authors employed dynamic dwell timers for VHO. Other works considered certain channel characteristics in their adaptive VHO schemes. Most of the authors, however, were concerned with all-radio HetNets. In the following, we present a review of these related works. Ylianttila et al. [10] demonstrated the need for an adaptive dwell timer that can respond to variations in system conditions. In addition, they suggested that such a timer should have a finite value. In their work, Lee et al. presented an adaptive dwell timer whose value was varied according to the utilities of current and target base stations (BSs) [11]. Furthermore, the value of their dwell timer was influenced by the movement direction of mobile station (MS). A variable width dwell timer for 4G heterogeneous networks was proposed by Haider et al. [12]. They suggested varying the dwell duration according to the speed of mobile terminals (MTs). Similarly, Qiu et al. adjusted the value of their dwell timer according to MT's speed before triggering handover [13]. Madaan et al. incorporated a dynamic dwell timer into their $\mathrm{VHO}$ algorithm [14]. In their case, the value of timer was not only influenced by speed of MS but also by the change in its direction and the potential for a ping-pong handover [15]. To avoid call dropping during communication in WiMAX networks, Sridevi et al. introduced a dwell timer in BSs to prevent ping-pong handovers [16]. Smaoui et al. proposed the dwell timer concept in their VHO decision algorithm to minimize handoff costs by reducing unnecessary and unbeneficial handoffs [17]. To ensure the proper operation of handover decision parameters, $\mathrm{Li}$ et al. adjusted the timeto-trigger (TTT) according to the speed of MT. In the work of Liang et al., the threshold for initiating handovers was adaptive to the application quality of service (QoS) requirements, error resilience, and channel bit error rate [18].

Most of the dwell timers employed in some of the VHO schemes discussed above are only sensitive to some characteristics of user mobility. Moreover, the nature of interruptions in VLC HetNets is different from that of all-radio HetNets; hence, the dwell timers employed in the preceding works cannot be applied directly to VLCWiFi HetNets. The interruptions in all radio HetNets last for several seconds and are difficult to detect due to fading, path loss, and signal fluctuations. On the other hand, the interruptions in VLC HetNets can last for a few seconds and are easily detected since received signal strength (RSS) degrades sharply when VLC channel is blocked. Wang et al. suggested that the dwell time, before handover decision, when VLC-LoS link is interrupted be adjusted based on system conditions to optimize the performance of a D-VHO scheme in VLC-RF HetNets [19]. Subsequently, Liu et al. proposed a dynamic dwell timer based on the prediction of time interval for MT to cross VLC/WLAN boundary [20]. However, the effect of physical obstructions between MT and VLC-LoS link could lead to wrong predictions. Earlier on, Hou et al. proposed a fuzzy logic-based VHO scheme that combines the merits of I-VHO and static D-VHO schemes using the failure probability of a VHO to radio channel as one of the input metrics [9]. Liang et al. also proposed a VHO algorithm based on prediction that relies on VLC channel interruption durations, message sizes, and access delays as input metrics for proper handover decision [21].

Recently, Yan et al. proposed a handover strategy for a combined VLC and WiFi system that infers whether a disruption in VLC link will last long or not, based on the quality of VLC signal, the MT's wireless coverage and loading condition, and decides whether to switch to $\mathrm{WiFi}$ or not [22]. A fuzzy logic (FL)-based dynamic handover scheme has been proposed by Wang et al. in which the average signal-to-interference-plus-noise ratio (SINR) of Light Fidelity (LiFi) channel is applied as an input metric to ascertain the transiency of LoS link blockage and appropriately assign users to either RF or LiFi access point (AP) [23]. Saud et al. presented a fast handover scheme for hybrid optical-RF systems that efficiently monitors the VLC link and switches quickly to RF when there is link failure. The VLC link is monitored and handover decision is made in between network and data-link layers [24]. In [7], we proposed a handover protocol for VLC HetNets whereby MT sets a dwell time to avoid frequent switching to RF network and service termination. Unnecessary handovers to RF network were avoided when the value of dwell timer exceeded the period for MT to cross the overlapped area. A VHO algorithm based on multiple attributes for a hybrid VLC-Femto system was presented by Liang et al. They considered dynamic network parameters such as average VLC interruption duration and VLC total bit rate to assess the abilities of candidate VHO decisions to handle VLC signal blockage or overload [25]. Most of the VHO schemes proposed for VLC HetNets 
have to decide whether to switch to RF or not when VLC channel is interrupted. However, excessive delays can arise if VLC link interruption is long and VHO to RF is not executed. Besides, most of the input parameters for these VHO schemes are difficult to obtain in real time. On the other hand, a few authors have applied the idea of dwell timing to VHO in VLC HetNets. Since VLC network is the preferred network in a VLC-WiFi HetNet, the goal should be to keep the MT in VLC mode as long as possible and execute VHO to WiFi only when necessary.

Based on the issues above, a channel adaptive D-VHO (CAD-VHO) scheme is presented in this article. In this scheme, a dynamic dwell timer that responds to the trend of VLC channel availability is employed. The objective is to avoid unnecessary VHOs considering the history of VLC accessibility. To achieve this, the average duration of VLC channel being available and unavailable are used to vary the width of dwell timer. The main contributions of this paper are summarized as follows:

- We propose a dynamic D-VHO scheme that is adaptive to the rate of blocking and recovery of VLC channel.

- Our proposed CAD-VHO scheme can behave like an I-VHO scheme if the rate of recovery of VLC optical link is insignificant.

- The CAD-VHO scheme is sensitive to the effect of user mobility and surrounding objects on LoS optical channel availability.

- Simulation results show that our proposed scheme outperforms static D-VHO and I-VHO schemes by reducing the number of $\mathrm{VHOs}$ while achieving a higher average data rate in most cases.

\section{System model}

We consider a HetNet with a WiFi AP and a number of overlapping VLC hotspots in a room. The WiFi is assumed to be always available anywhere in the room. We assume that the VLC hotspots are mounted on the ceiling of the room with an array of LEDs as transmitters and also for illumination. The Lambertian optical source model is used in the VLC transmitter. It denotes that the brightness to an observer is the same regardless of the observer's angle of view. Therefore, the projection of a VLC hotspot on the ground can be regarded as a circle as shown in Fig. 1. Since the VLC transmitters use the same lightwave bandwidth, there is high co-channel interference in the overlapped area of VLC hotspots [26]. Therefore, the VLC link is assumed to be blocked in these areas. Additionally, there is a surrounding mobile object that can obstruct VLC-LoS channel. An MT with two wireless interfaces can use either VLC or WiFi for downlink transmission depending on their availability. Furthermore, the MT can change downlink transmission mode from VLC to WiFi and vice versa. For uplink transmission including sending handover requests, the MT uses WiFi only. Mobile users prefer VLC for downlink transmission due to its higher transmission rate. However, when VLC is not available, MT may decide to change to WiFi mode to maintain connectivity. In a dwell vertical handover (D-VHO) scheme, the multimode MT waits for a period of time before deciding to switch to WiFi mode when VLC link is blocked. In this scheme, an MT sends a request to the $\mathrm{VHO}$ controller to change its downlink transmission to WiFi only if VLC link is still blocked after the dwell time. The controller in the HetNet is responsible for choosing the transmission mode of downlink traffic. It receives handover requests from MTs via the WiFi AP to change the transmission path for downlink packets. There is a handover delay between the time of $\mathrm{VHO}$ request and the time of $\mathrm{VHO}$ execution [20]. The switching mechanism in each VLCWiFi node follows that in [3, 7]. In this work, our focus is on the amount of time for MT to wait when VLC channel is blocked before requesting for a $\mathrm{VHO}$ to WiFi network.

\section{Channel adaptive dwell vertical handover scheme}

In this section, we propose a channel adaptive dwell vertical handover scheme (CAD-VHO) in the VLC-WiFi HetNet. Analysis of VLC-LoS link blocking is initially presented. This is followed by the description of our dynamic dwell timer, and then the algorithm for the implement of our scheme is given.

\subsection{Blocking and non-blocking events}

Over a period of time, the VLC link can be lost and recovered in a recurring manner as shown in Fig. 2. When the VLC link is lost, a blocking event or unavailable session starts. On the other hand, if the link is recovered, then a non-blocking event or available session begins. An available session begins when an unavailable sessions ends and vice versa. As seen from Fig. 2, the duration of a blocking or non-blocking event is a random variable. The random movement of MTs will largely determine the duration of blocking or non-blocking of VLC link. For example, if the MT moves into the overlap area of the adjacent VLC hotspots or the non-coverage area of the VLC network, the VLC link will be terminated. For a given direction, a higher MT speed results in a shorter interruption since MT spends less time in the region of VLC link unavailability. Furthermore, for a given velocity, the longer the duration of movement the higher the frequency of LoS blocking since MT makes more transitions from VLC coverage. Also, the more an MT is stationary at one location the less the frequency of blocking and/or recovery of the LoS optical channel. If, for instance, MT moves into the overlap area of adjacent VLC hotspots and stops there 


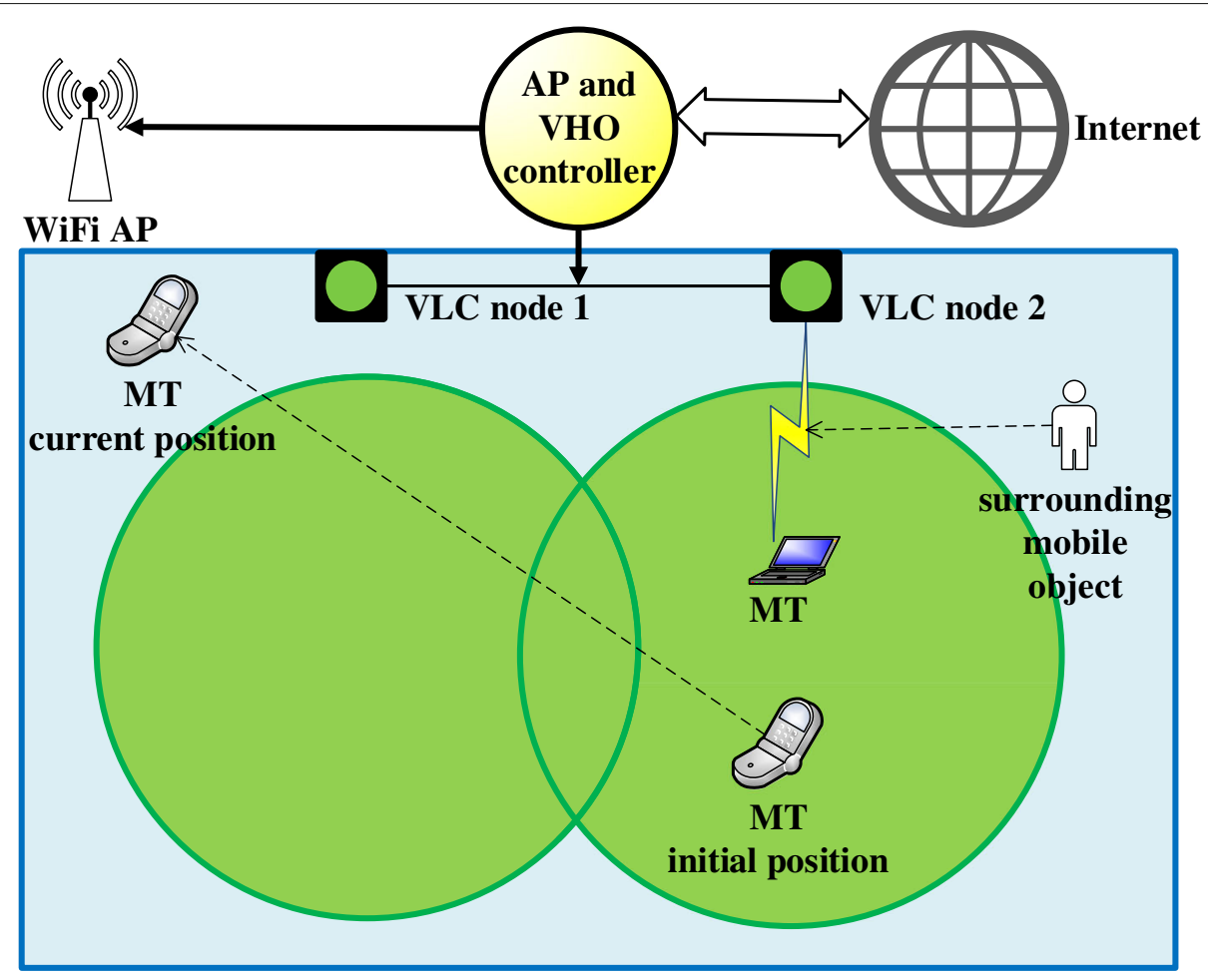

Fig. 1 The system model of VLC and WiFi heterogeneous network

for a long time, there will be a long interruption in LoS optical channel. On the other hand, if MT usually stays in VLC coverage for a long time, the frequency of blocking will be low. However, the availability or otherwise of VLC channel is not only as a result of user mobility. For example, a physical object such as a human can obstruct the LoS link between MT and VLC transmitter as shown in Fig. 1.

At the end of an available session, if the user is connected to VLC, it must decide whether to switch to WiFi or not. If the VLC-LoS blocking occurs frequently, it can lead to unnecessary handovers and the ping-pong effect. For a D-VHO scheme, a dwell time will be set in anticipation of link recovery to reduce unnecessary handovers. However, if the recovery time is greater than the dwell time, the unnecessary delay will largely degrade system

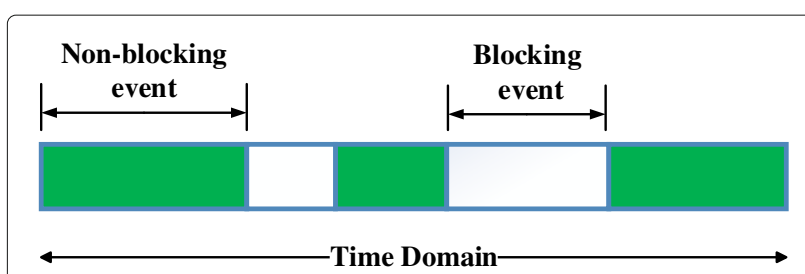

Fig. 2 Blocking and non-blocking events over a period of time performance. In our work, we utilize the average duration of blocking and non-blocking events, in the event of blocking of LoS optical link, to compute the dwell time. If the VLC link is still unavailable after the dwell time, handover is executed from VLC to WiFi; otherwise, MT remains connected to VLC. On the other hand, when the MT is connected to WiFi and an available session begins, the communication link is switched immediately to VLC.

\subsection{Channel adaptive dwell timer}

By using up-to-date information about the past events of blocking and unblocking of VLC channel, we formulate a dynamic dwell VHO timer. In this way, the historical information about the availability or unavailability of VLC channel relative to a particular user is factored into the dwell VHO scheme. Let $\gamma_{1}$ and $\gamma_{2}$ be the mean duration of VLC link being available and unavailable, at a particular instance, respectively. Then the rate of VLC channel changing from being lost to recovered and vice versa is given as $1 / \gamma_{2}$ and $1 / \gamma_{1}$ respectively [19]. The probability $P_{r}$ of recovering VLC channel just after being blocked is given by

$$
P_{r}=\frac{\gamma_{1}}{\gamma_{1}+\gamma_{2}}
$$


Consequently, the probability $P_{b}$ of VLC channel remaining blocked is given as

$$
P_{b}=\frac{\gamma_{2}}{\gamma_{1}+\gamma_{2}}
$$

At the instance of blocking of VLC channel, the dwell time $t$ is computed as

$$
t= \begin{cases}t_{\max } \times\left(1-\frac{\gamma_{2}}{\gamma_{1}+\gamma_{2}}\right) & \text { if } N_{B}>0 \\ t_{\max } & \text { if } N_{B}=0\end{cases}
$$

where $t_{\max }$ is the maximum value of dwell timer and $N_{B}$ is the number of blocking events before the current event. The choice of $t_{\max }$ is very crucial to the performance of the CAD-VHO scheme. The optimum value for $t_{\max }$ depends on the simulation scenario. Besides, a higher value for $t_{\max }$ can result in less number of handovers and can be tolerated by non-real-time applications. However for real-time applications, there is the need to limit the maximum value of dwell timer. In this work, we choose $t_{\max }$ from multiples of conventional dwell time values and perform simulation to find the optimum value. The mean non-blocking and blocking periods, $\gamma_{1}$ and $\gamma_{2}$, will be evaluated by the dwell time algorithm incorporated into MT.

Equation (3) indicates that the lower the recovery probability $P_{r}$ of VLC link, the lower the dwell time set. This is an informed decision, since it takes a longer time for VLC link to be recovered. Therefore, additional dwelling is likely to degrade system performance. On the other hand, the higher the recovery probability $P_{r}$ of VLC OW link, the higher the dwell time since LoS link is known to be blocked for a relatively shorter period of time. Therefore, we can infer that VLC link will be recovered in a shorter period of time and, in this way, we can avoid unnecessary handover. It is worth noting that as the probability of VLC link remaining blocked approaches $1\left(P_{b} \rightarrow 1\right)$, our proposed dwell timer tends to trigger $\mathrm{VHO}$ early. $\mathrm{VHO}$ is triggered almost immediately when $t \approx 0$. Therefore, our proposed channel adaptive dwell timer can initiate an I$\mathrm{VHO}$ or D-VHO depending on the rate of blocking and recovery of VLC channel. The dwell timer proposed is different from that in [20] in the following ways:

- The value of our dwell timer is adjusted according to the recovery probability of VLC link while the VLC/WLAN boundary crossing intervals are used to adjust the value of their dwell timer.

- Our timer's value is influenced by the rate of blocking and recovery of VLC link while their timer value is influenced by the frequency of crossing VLC/WLAN boundary.
- Our timer's value is affected by user mobility and surrounding objects while the value of their timer is affected only by user mobility.

- Our proposed timer has a maximum value while theirs uses a baseline value.

- The threshold of our dwell timer is adjusted using up-to-date information on VLC link availability while the threshold of their dwell timer is updated according to latest predicted crossing interval.

\subsection{Dwell time algorithm}

In the situation where an MT is connected to a VLC hotspot and the link suddenly becomes blocked, a dwell time will be set for that instance. The following CADVHO algorithm is proposed to calculate the dwell time, before handover decision, when VLC becomes unavailable. In the algorithm, VLC_State is an in-built function in MT which indicates the availability of VLC channel. When VLC_State $=1$, it means VLC channel is available. However, when VLC_State $=0$, it indicates that VLC channel is unavailable. $V_{n}$ and $V_{b}$ represent the number of nonblocking and blocking events respectively. To be able to keep track of the history of VLC accessibility, the algorithm records the time the last interruption or resumption occurred. $\Delta t_{i-s}$ is the time interval between time steps $i$ and $s$, used to determine the period between the current blocking or non-blocking event and the previous one as illustrated in Figs. 3 and 4.

All the historical information needed is captured in the proposed CAD-VHO algorithm and the calculation of the dwell time is relatively less complex. When the VLC link resumes, the mean duration of link interruption is recalculated and the number of blocking events is increased by $1\left(V_{b}+1\right)$. In the same way, when the VLC link is blocked, the mean duration of LoS optical channel being available is recalculated and the number of available sessions is increased by $1\left(V_{b}+1\right)$. Our dwell time algorithm is unlike that proposed in [20] in the following ways:

- It records the time of VLC link interruption and recovery while the other algorithm records the VLC/WLAN boundary crossing time interval.

- Our algorithm calculates the mean VLC link blocking and non-blocking durations when link is recovered

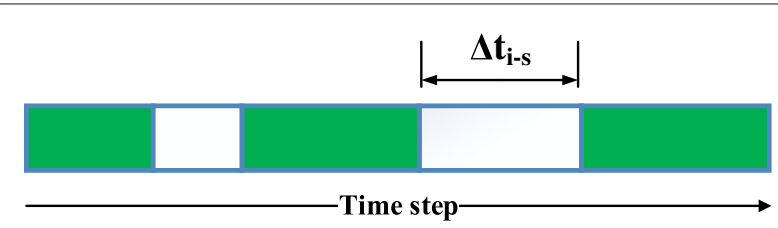

Fig. 3 Illustration of the time interval $\Delta t_{i-s}$ between current non-blocking event and the previous one 


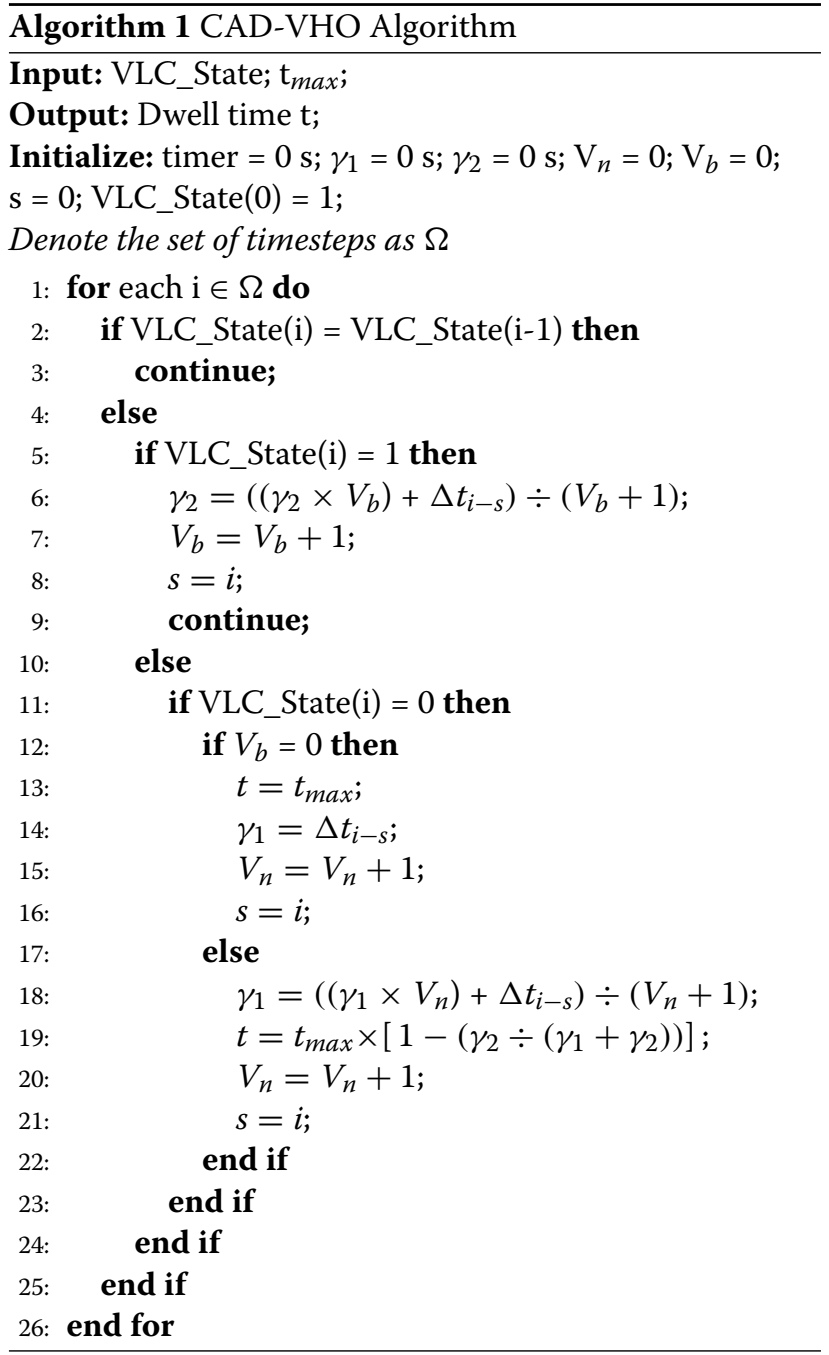

and lost respectively. However in [20], the algorithm updates predicted time interval whenever MT crosses WLAN/VLC boundary.

- In our algorithm, dwell time is computed only when VLC link is blocked or interrupted. On the contrary, the other algorithm computes dwell time whenever MT crosses WLAN/VLC boundary.

- CAD-VHO algorithm does not apply any weighting factors while the other algorithm applies weighting factors to compute dwell time.

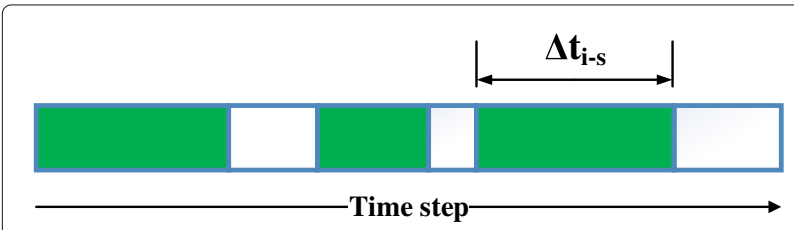

Fig. 4 Illustration of the time interval $\Delta t_{i-s}$ between current blocking event and the previous one
In the following section, we perform simulation to obtain the optimum value for $t_{\max }$ and compare our scheme with benchmark designs.

\section{Simulation}

Simulation is carried out using MATLAB to find the optimum value of $t_{\max }$ and to compare the performance of CAD-VHO scheme with that of conventional I-VHO and D-VHO schemes [19] based on the average number of VHOs and average data rate. In I-VHO, the transmission mode is switched to Wi-Fi as soon as VLC link is interrupted. The D-VHO scheme sets a fixed waiting time $\left(t_{s}=0.5 \mathrm{~s}, t_{s}=1 \mathrm{~s}\right)$ before handover decision in the event of VLC link blocking. In this scheme, transmission mode is switched to Wi-Fi only when the VLC link recovery time is greater than the waiting time $t_{s}$.

The simulation scenario is set up as a room with nine uniformly arranged VLC hotspots and a WiFi AP. The room has a height of $5 \mathrm{~m}$. The VLC LEDs have a semiangle of irradiance of $17^{\circ}$ and the VLC receivers have a field of view (FOV) of $60^{\circ}$ [27]. The area of the floor in the room is $64 \mathrm{~m}^{2}$, and each VLC hotspot covers an area of $2.25 \pi \mathrm{m}^{2}$. The coverage of each VLC hotspot is a circle centered at $(1.5,1.5),(4,1.5),(6.5,1.5),(1.5,4)$, $(4,4),(6.5,4),(1.5,6.5),(4,6.5)$, and $(6.5,6.5)$. WiFi is accessible anywhere in the room. We assume that the data rates of both WiFi and VLC are equal everywhere in their coverage region [20]. The coverage areas of adjacent VLC hotspots intersect to form overlap areas. An overlap area is an overlapped-illumination area of two or more VLC LEDs. Due to the strong interference of existing optical signals from adjacent VLC transmitters in an overlap area, it is difficult for the VLC receiver to recover the transmitted signal [28]. Therefore, the VLC link from the associated VLC node is assumed to be terminated in the overlap areas of VLC hotspots. The simulation scenario is illustrated in Fig. 5. There are two types of mobile terminals (MTs) according to behavior: a restless MT and a stationary MT both initially connected to VLC. The restless MT undergoes random movements during the simulation. The direction of movement is assumed to be a uniform random variable within 0 and $2 \pi$ radians [29]. At every point in time, we determine whether restless MT is in VLC coverage or not. In addition, we estimate the mean duration of staying in and out of VLC coverage. Anytime the MT moves out of or into VLC coverage area, the mean blocking and non-blocking durations are updated. Our algorithm utilizes this information to calculate the dwell time before making the handover decision. During the simulation, the stationary MT in VLC coverage experiences occasional VLC-LoS link interruption by a surrounding mobile object. The duration of blocking and recovery of the VLC channel are assumed to be exponential random variables with the rate of recovery and 


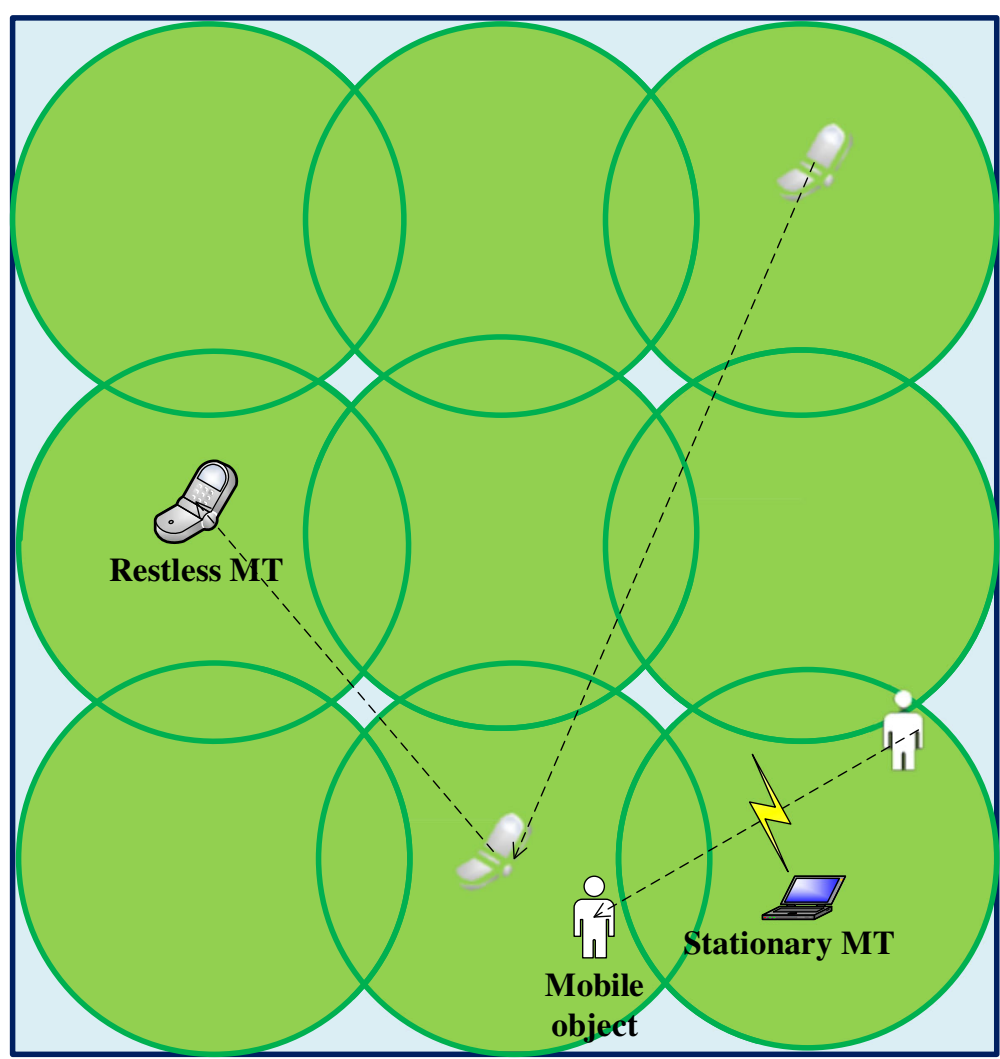

Fig. 5 The simulation scenario for performance evaluation

blocking as parameters respectively [19]. As in the case of the restless MT, the mean blocking and non-blocking periods are estimated and dwell time is calculated by the CAD-VHO algorithm whenever optical link blocking occurs before VHO decision. The handover delay, which is the period between $\mathrm{VHO}$ request and completion, is assumed to be normally distributed with a mean value and a variance [9].

In order to evaluate the performance of CAD-VHO using different values of $t_{\max }$, we set the velocity $v$, movement duration $m$, and pause time interval $p$ of restless MT as uniform random variables within the intervals stated in Table 1. Velocity of MT is defined as the speed of movement in a particular direction, movement duration is the time it takes for an MT at rest to move to another point, and pause time interval is the duration of staying at one point. The optimum value of $t_{\max }$ is chosen in order to compare the performance of CAD-VHO with the benchmark schemes (I-VHO and D-VHO). We evaluate the effect of the surrounding mobile object on the number of VHOs and data rate for I-VHO, D-VHO, and CAD-VHO schemes. The rate of blocking is fixed at $0.1 \mathrm{~s}^{-1}$ while the rate of recovery is increased from 0.3 to $1.2 \mathrm{~s}^{-1}$. The effects of mobility parameters $v, m$, and $p$ on the number
Table 1 Simulation parameters

\begin{tabular}{ll}
\hline Parameter & Value \\
\hline Room dimension & $8 \mathrm{~m}$ by $8 \mathrm{~m}$ by $5 \mathrm{~m}$ \\
Number of VLC hotspots & 9 \\
Coverage radius of each VLC hotspot & $1.5 \mathrm{~m}$ \\
Velocity of restless MT $v$ & $0.3-0.7 \mathrm{~ms}^{-1}$ \\
Movement duration of restless MT $m$ & $2-11 \mathrm{~s}$ \\
Pause time interval of restless MT $p$ & $3-12 \mathrm{~s}$ \\
Direction of restless MT's motion $d$ & $0-2 \pi$ radians \\
Rate of blocking of VLC channel by mobile object & $0.1 \mathrm{~s}^{-1}$ \\
Rate of recovery of VLC channel by stationary MT & $0.3-1.2 \mathrm{~s}^{-1}$ \\
Data rate of VLC & $1 \mathrm{Gbps}$ \\
Data rate of WiFi & $200 \mathrm{Mbps}$ \\
Mean handover delay & $0.4 \mathrm{~s}$ \\
Handover delay variance & $0.1 \mathrm{~s}$ \\
Static dwell time $t_{\mathrm{s}}$ & $1,0.5 \mathrm{~s}$ \\
Simulation period & $3600 \mathrm{~s}$ \\
Number of iterations & 2000
\end{tabular}


of $\mathrm{VHOs}$ and data rate for I-VHO, D-VHO, and CADVHO schemes are evaluated. To ascertain the effect of a mobility parameter, it is fixed at certain values with uniform intervals while the other mobility parameters are set as uniform random variables within the intervals given in Table 1. Each evaluation is performed for a large number of iterations to determine the average performance.

The number of VHOs for all schemes is initially set to zero for each iteration of the simulation process. In the case of I-VHO scheme, the number of VHOs is increased by 1 each time MT is transmitting via VLC and LoS link blocking occurs. For D-VHO $t_{s}=0.5 \mathrm{~s}$ and D-VHO $t_{s}=1 \mathrm{~s}$, the number of VHOs is increased by 1 when the duration of blocking is greater than $0.5 \mathrm{~s}$ and $1 \mathrm{~s}$ respectively. In the case of CAD-VHO, the number of VHOs is increased by 1 when the duration of blocking is greater than dwell time $t$. For all the schemes, when MT is transmitting via WiFi and VLC link is recovered, the number of VHOs is increased by 1 since $\mathrm{VHO}$ is executed immediately. The average number of $\mathrm{VHOs}$ is calculated by

$$
A_{V H O}=\frac{\sum_{k=1}^{N_{i}} N_{V H O}(k)}{N_{i}}
$$

where $A_{V H O}$ is the average number of VHOs for a particular VHO scheme, $N_{V H O}(k)$ is the number of VHOs for iteration $k$ and $N_{i}$ is the number of iterations. The average number of VHOs is directly proportional to the average total VHO signaling cost. We assume that the signaling cost of executing a VHO is constant. Therefore, a reduction in the number of VHOs by $\mathrm{c} \%$ results in a $\mathrm{c} \%$ reduction in the total VHO signaling cost.

To estimate the average data rate, we determine the transmission mode of MT, i.e., whether it is transmitting via VLC or WiFi. In addition, we obtain the time duration of being connected to VLC or WiFi. The average data rate is calculated by

$$
A_{D R}=\frac{\sum_{k=1}^{N_{i}} \sum_{s=1}^{N_{c}(k)} D R(c, k)^{*} T_{c}(k)}{\sum_{k=1}^{N_{i}} T_{i}(k)}
$$

where $A_{D R}$ is the average data rate for a particular VHO scheme, DR $(\mathrm{c}, \mathrm{k})$ is the data rate during the $c^{\text {th }}$ connection of iteration $k, T_{c}(k)$ is the duration of the $c^{\text {th }}$ connection of iteration $k, N_{c}(k)$ is the number of connections in iteration $k$, and $T_{i}(k)$ is the total time duration of iteration $k$.

\section{Results and discussion}

This section is made up of two parts. To begin with, we evaluate the performance of our scheme using different values of $t_{\max }$ to select the optimum value. Afterwards, we compare the performance of CAD-VHO scheme with that of conventional I-VHO and D-VHO schemes.

\subsection{Comparison between different maximum dwell durations}

In this section, we evaluate the performance of our algorithm using different values of $t_{\max }$. Each value of $t_{\max }$ results in a different timer and, hence, a different subscheme as shown in Table 2. The evaluation is based on the number of VHOs and average data rate of restless MT.

As $t_{\max }$ increases from 1 to $2 \mathrm{~s}$, the number of $\mathrm{VHOs}$ is decreased by about $50 \%$. Increasing $t_{\max }$ from 2 to $3 \mathrm{~s}$ and from 3 to $4 \mathrm{~s}$, results in the decrement of the number of VHOs by $24 \%$ and $10 \%$ respectively. The results in Fig. 6 show that when $t_{\max }$ increases from 1 to $2 \mathrm{~s}$, the number of VHOs is reduced drastically. However, as $t_{\max }$ increases beyond $2 \mathrm{~s}$, there is relatively marginal reduction in the number of $\mathrm{VHOs}$ because the duration of most interruptions is greater than $1 \mathrm{~s}$ but less than $2 \mathrm{~s}$. Furthermore, the decrement in the number of VHOs is even more marginal as $t_{\max }$ increases from 3 to $4 \mathrm{~s}$. This means that there is a certain value for $t_{\max }$ beyond which the number of VHOs remains virtually the same since the duration of blocking has a certain average maximum value. Figure 7 shows that when $t_{\max }$ increases from 1 to $2 \mathrm{~s}$, there is an improvement in average data rate by $5 \mathrm{Mbps}$. On the contrary, as $t_{\max }$ increases beyond $2 \mathrm{~s}$ there is degradation in average data rate by $0.9 \mathrm{Mbps}$. Furthermore, the extent of degradation is even more severe as $t_{\max }$ increases from 3 to $4 \mathrm{~s}$, decreasing by $3 \mathrm{Mbps}$. The decrement in average data rate can be attributed to the reduction in the number of necessary handovers as $t_{\max }$ increases beyond $2 \mathrm{~s}$. This means that there is a certain value for $t_{\max }$ beyond which any increment is detrimental because a longer waiting time can degrade the data rate offered to MT if there is a long interruption in VLC channel.

In order to compare our scheme with benchmark designs, we need to choose the optimum value of $t_{\max }$. We choose $2 \mathrm{~s}$ as our optimum value for the following reasons:

- It produces the highest average data rate, hence most suitable for most Internet applications.

- Compared with $t_{\max }$ less than $2 \mathrm{~s}$, the VHO signaling cost is reduced by at least $50 \%$.

- It is more suitable for real-time applications than $t_{\max }$ greater than $2 \mathrm{~s}$ since long dwelling can result in excessive delay.

Table 2 CAD-VHO with different maximum dwell durations

\begin{tabular}{lll}
\hline$t_{\max }(\mathrm{s})$ & Timer & Sub-scheme \\
\hline 1 & $\mathrm{~T} 1$ & CAD-VHO-T1 \\
2 & $\mathrm{~T} 2$ & CAD-VHO-T2 \\
3 & $\mathrm{~T} 3$ & CAD-VHO-T3 \\
4 & $\mathrm{~T} 4$ & CAD-VHO-T4 \\
\hline
\end{tabular}




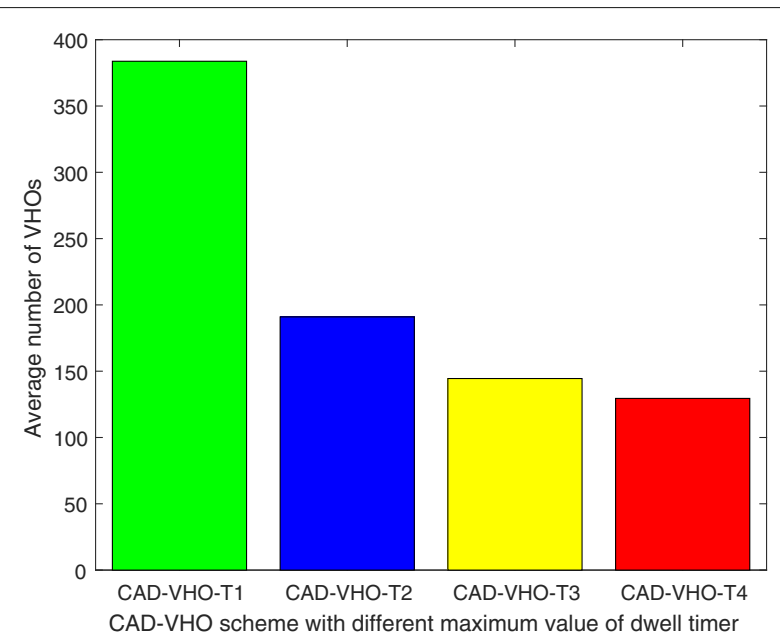

Fig. 6 Simulation results of the average number of VHOs for the CAD-VHO scheme with different maximum dwell durations $t_{\max }$

\subsection{Comparison with benchmark schemes}

In this part, we evaluate the performance of our scheme and that of the benchmarks. We first evaluate the effect of the rate of recovery of VLC link by stationary MT on the number of VHOs for the various schemes. Given the same simulation period, there are more interruptions when the rate of recovery increases because the interruptions last for shorter periods. Therefore, I-VHO results in more VHOs but D-VHO and CAD-VHO avoid more VHOs since their dwell times increasingly exceed the VLC-LoS recovery time. However, $\mathrm{CAD}-\mathrm{VHO}$ reduces the number of VHOs by about $80 \%$ and $64 \%$ when compared to DVHO $t_{s}=0.5 \mathrm{~s}$ and D-VHO $\mathrm{t}_{s}=1 \mathrm{~s}$ schemes respectively. The results in Fig. 8 show that our scheme has the lowest VHO signaling cost due to its optimum maximum dwell

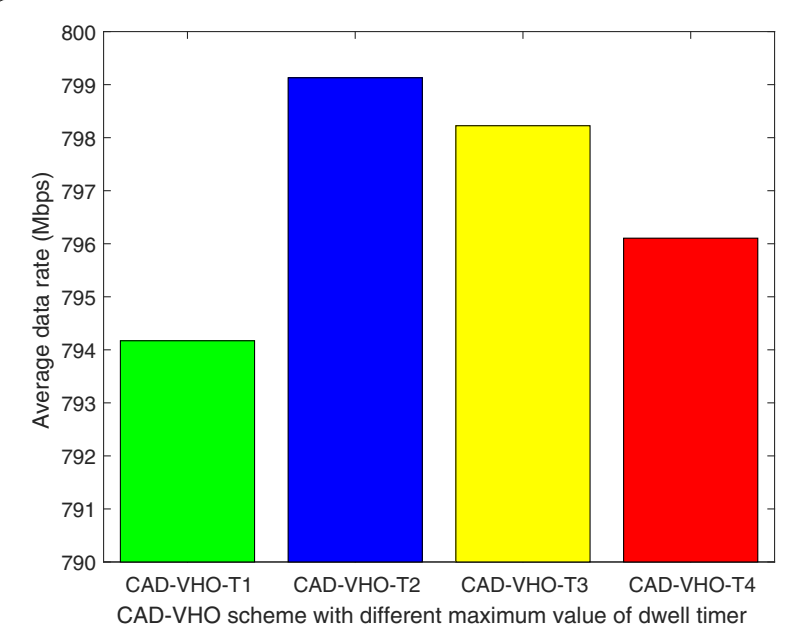

Fig. 7 Simulation results of the average data rate for the CAD-VHO scheme with different maximum dwell durations $t_{\text {max }}$ time and ability to adjust the waiting time before handover decision according to the rate of blocking and recovery of VLC channel.

Secondly, the effect of velocity $v$ of restless MT on the number of VHOs for the various schemes is evaluated. As $v$ increases from 0.3 to $0.7 \mathrm{~ms}^{-1}$, the average number of $\mathrm{VHOs}$ for CAD-VHO constantly reduces from 218 to 149. This inverse relationship exists because the blocking events increasingly last for shorter periods and therefore the dwell time increasingly exceeds the VLC-LoS recovery time as velocity increases. For $v=0.3 \mathrm{~ms}^{-1}$, CAD-VHO scheme reduces the number of VHOs by about $47 \%$, $34 \%$ and $20 \%$ when compared to I-VHO, D-VHO $t_{s}=0.5 \mathrm{~s}$ and $\mathrm{D}-\mathrm{VHO} t_{s}=1 \mathrm{~s}$ schemes respectively. At $v=0.7$ $\mathrm{ms}^{-1}$, CAD-VHO scheme reduces the number of VHOs by about $82 \%, 70 \%$, and $32 \%$. The results in Fig. 9 show that our scheme has the lowest VHO signaling cost irrespective of the velocity of MT. This advantage is even more pronounced as velocity increases because unlike the other schemes, the CAD-VHO scheme is adaptive to the increasing rate of blocking of optical channel due to increasing velocity.

Next, we ascertain the effect of movement duration $m$ of restless $\mathrm{MT}$ on the number of VHOs for the various schemes. Longer movement duration means higher rate of blocking due to more transitions from VLC coverage. As movement duration $m$ of MT increases from 2 to $11 \mathrm{~s}$, the number of VHOs for CAD-VHO is increased marginally from 145 to 187 . This shows that our scheme is not vulnerable to the movement duration of MT since it adjusts the dwell time according to the effect of movement duration on the rate of blocking of VLC channel. For $m=2 \mathrm{~s}$, CADVHO scheme reduces the number of VHOs by about $52 \%$, $35 \%$, and $13 \%$ when compared to I-VHO, D-VHO $t_{s}=$ $0.5 \mathrm{~s}$ and D-VHO $t_{s}=1 \mathrm{~s}$ schemes respectively. At $m=$ $11 \mathrm{~s}, \mathrm{CAD}-\mathrm{VHO}$ scheme reduces the number of VHOs by about $75 \%, 64 \%$, and $41 \%$ when compared to I-VHO, $\mathrm{D}-\mathrm{VHO} t_{s}=0.5 \mathrm{~s}$ and D-VHO $t_{s}=1 \mathrm{~s}$ schemes respectively. The results in Fig. 10 indicate that our scheme has the lowest VHO signaling cost irrespective of the movement duration of MT. This advantage is even more visible as movement duration increases due to the fact that the other schemes are static while CAD-VHO is responsive to the effect of movement duration on the LoS optical link blocking.

Additionally, we investigate the effect of pause time interval $p$ of restless MT on the number of VHOs for the various schemes. As $p$ increases from 3 to $12 \mathrm{~s}$, the number of VHOs for CAD-VHO is decreased from 255 to 132. Generally, the number of VHOs for all the schemes is reduced as pause time interval of MT increases since the rate of blocking is reduced considerably. For all values of $p, \mathrm{CAD}-\mathrm{VHO}$ scheme reduces the number of VHOs by about $72 \%, 60 \%$, and $36 \%$ when compared to I-VHO, 


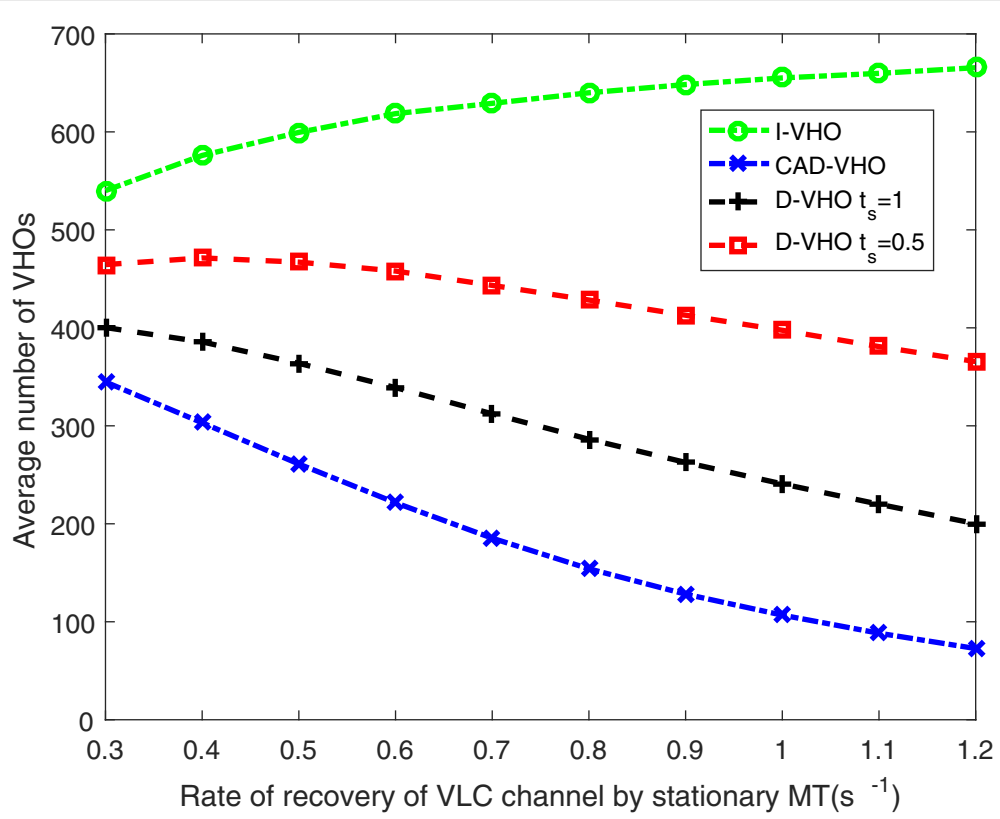

Fig. 8 Simulation results of the average number of VHOs against the rate of recovery of VLC channel

$\mathrm{D}-\mathrm{VHO} t_{s}=0.5 \mathrm{~s}$, and D-VHO $t_{s}=1 \mathrm{~s}$ schemes respectively. The results in Fig. 11 reveal that our scheme has the lowest VHO signaling cost irrespective of the pause time interval of MT since it minimizes unnecessary VHOs by setting an optimum waiting time considering the rate of blocking and recovery of VLC channel due to pause time interval.
The effect of the rate of recovery of optical channel by stationary MT on the average data rate is investigated in Fig. 12. As the rate of recovery of optical channel increases from 0.3 to $1.2 \mathrm{~s}^{-1}$, the average data rate constantly increases for all the schemes. This shows that a higher rate of VLC-LoS link recovery has a positive effect on data rate because of shorter interruptions in VLC channel.

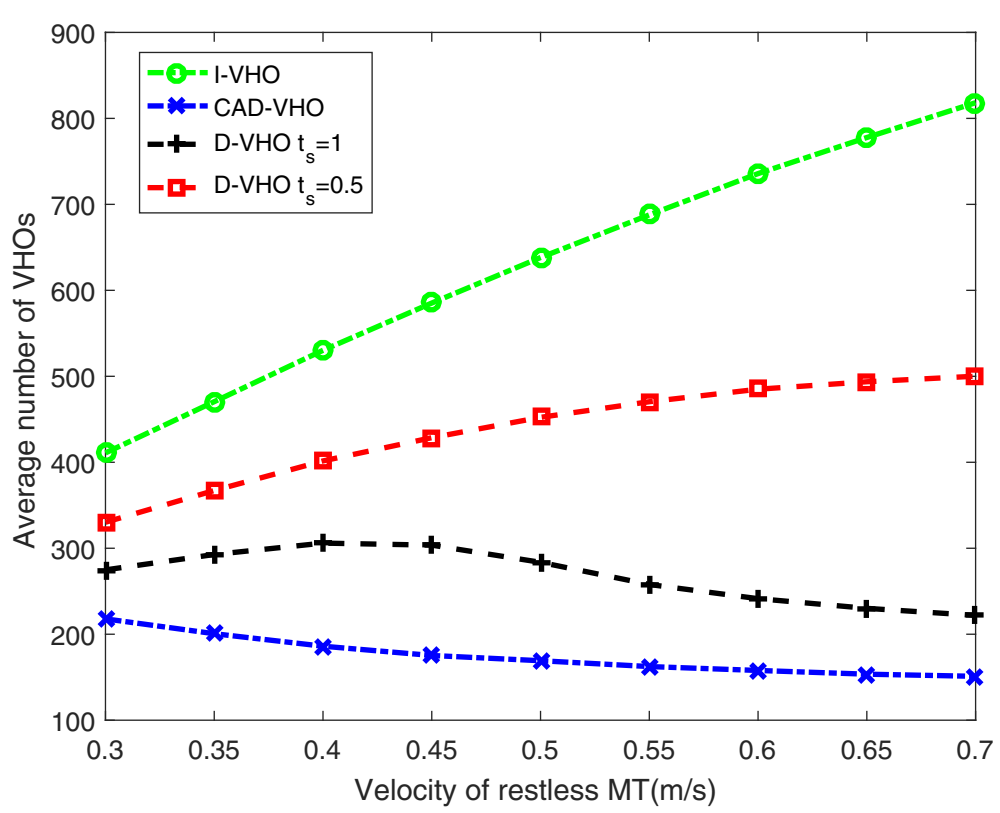

Fig. 9 Simulation results of the average number of VHOs against velocity of restless MT 


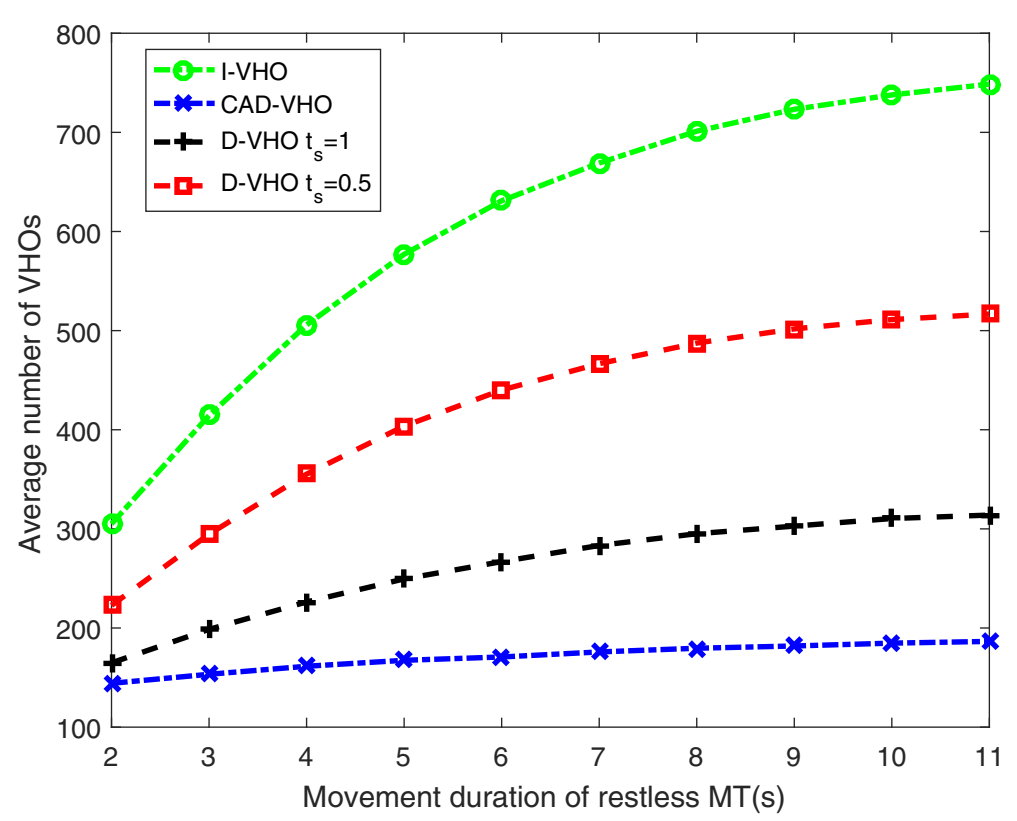

Fig. 10 Simulation results of the average number of VHOs against movement duration of restless MT

The average data rate for CAD-VHO is increased by 157.3 Mbps. Meanwhile, that of I-VHO, D-VHO $t_{s}=0.5 \mathrm{~s}$, and D-VHO $t_{s}=1 \mathrm{~s}$ is increased by $130.9 \mathrm{Mbps}, 143.3 \mathrm{Mbps}$, and 151.1 Mbps respectively. I-VHO has the highest data rate when the rate of recovery of optical channel is less than $0.5 \mathrm{~s}^{-1}$ since the probability for long interruptions is relatively high. The results in Fig. 12 show that our scheme has the highest average data rate as the rate of recovery of optical channel increases beyond $0.5 \mathrm{~s}^{-1}$ since CAD-VHO avoids more unnecessary VHOs than the other schemes by increasing its dwell time.

We investigate the effect of velocity $v$ of restless MT on the average data rate for the various schemes. Higher velocity means higher frequency of blocking and shorter interruptions of optical link. Unnecessary VHO switches downlink transmission to the lower data rate WiFi channel

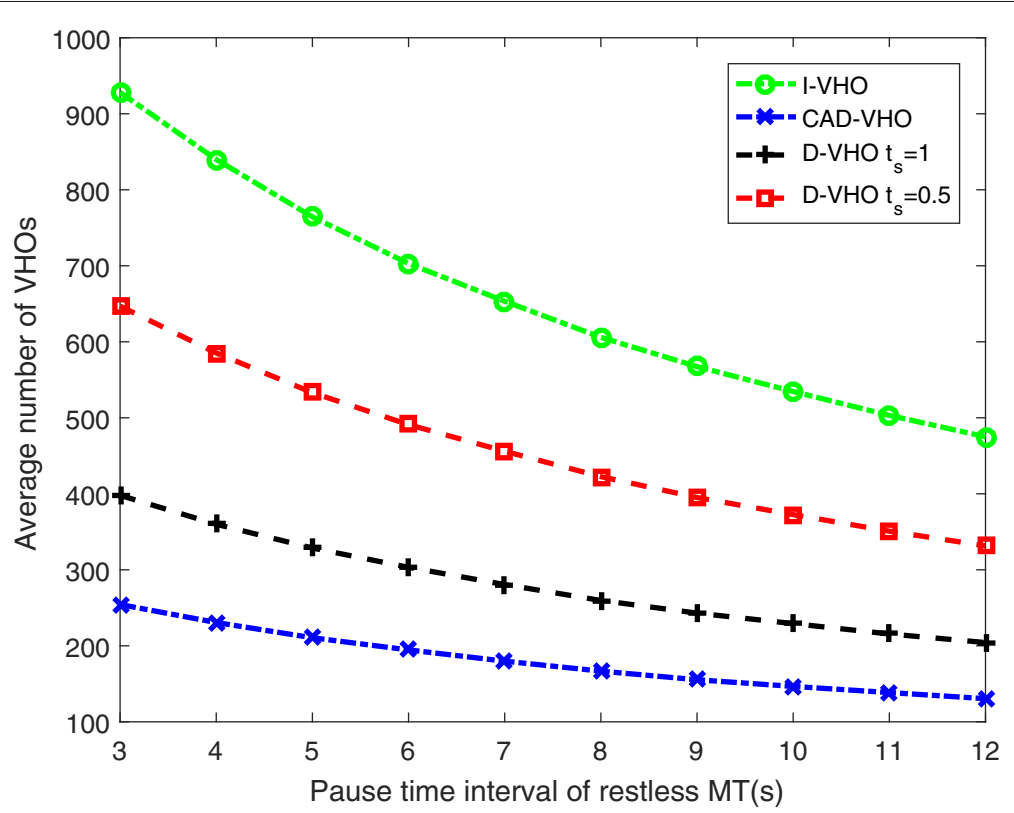

Fig. 11 Simulation results of the average number of VHOs against pause time interval of restless MT 


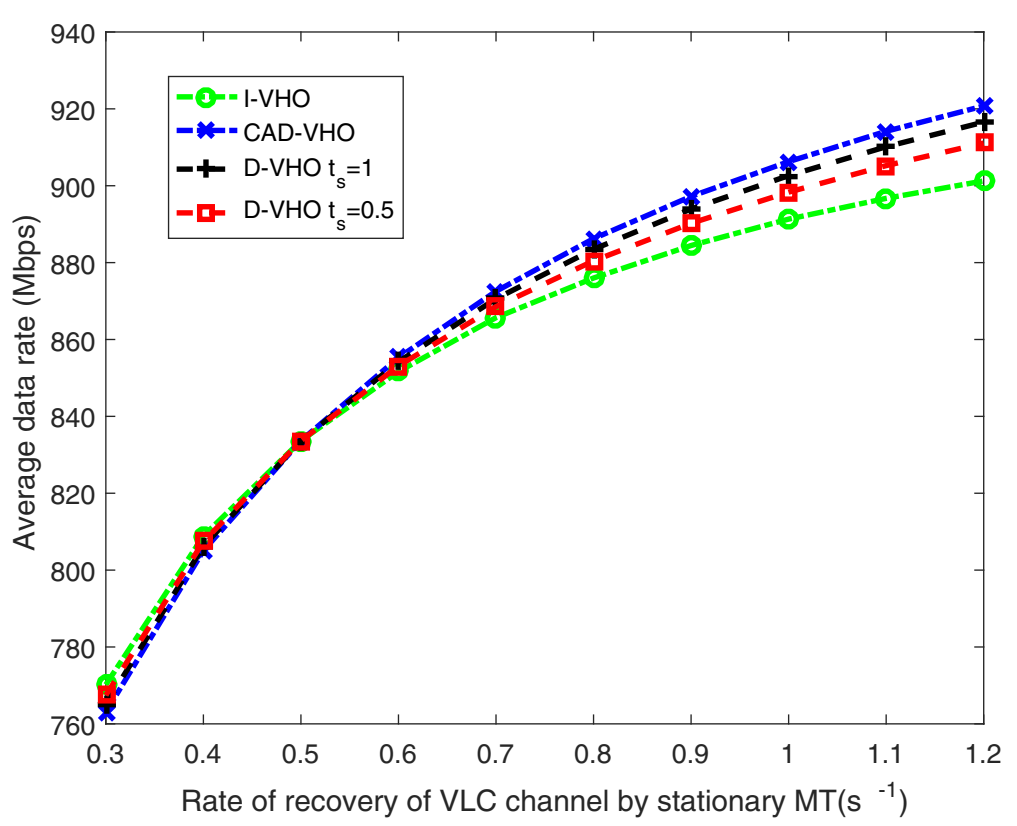

Fig. 12 Simulation results of the average data rate against rate of recovery of VLC channel

when a short interruption occurs. As $v$ increases from 0.3 to $0.7 \mathrm{~ms}^{-1}$, the average data rate for CAD-VHO is relatively stable especially when compared with I-VHO and $\mathrm{D}-\mathrm{VHO} t_{s}=0.5 \mathrm{~s}$. This indicates that our scheme is least vulnerable to the velocity of MT as it considers the effect of velocity on the rate of blocking of VLC channel and reduces unnecessary $\mathrm{VHOs}$ accordingly. The average data rate for CAD-VHO ranges between 801.3 and $800.3 \mathrm{Mbps}$ while that of I-VHO ranges between 804 and $780.8 \mathrm{Mbps}$. For D-VHO $t_{s}=0.5 \mathrm{~s}$ and D-VHO $t_{s}=1 \mathrm{~s}$, the average data rate ranges between 803.2 and $789 \mathrm{Mbps}$ and between 802.1 and 799.2 Mbps respectively. Figure 13 shows that our scheme has the highest average data rate as velocity $v$ increases beyond $0.35 \mathrm{~ms}^{-1}$ since the number of unnecessary VHOs of the other schemes increases while that of CAD-VHO reduces steadily. However, the average data rate for D-VHO $t_{s}=1 \mathrm{~s}$ increases beyond $v=0.5 \mathrm{~ms}^{-1}$ since the number of unnecessary VHOs begins to reduce owing to the increasing probability of a short interruption less than $1 \mathrm{~s}$.

Next, we ascertain the effect of movement duration $m$ of restless MT on the average data rate for the various schemes. As $m$ increases from 2 to $11 \mathrm{~s}$, the average data rate for all the schemes constantly decreases. This shows the negative impact of movement duration on data rate since the rate of blocking increases as $m$ increases. However, this impact is least severe for CAD-VHO as it reduces unnecessary VHOs by increasing the dwell time when VLC channel interruption occurs. The average data rate for CAD-VHO is reduced by $11.5 \mathrm{Mbps}$. Meanwhile, that of I-VHO, D-VHO $t_{s}=0.5 \mathrm{~s}$ and D-VHO $t_{s}=1 \mathrm{~s}$ is reduced by $23.4 \mathrm{Mbps}$, 20.6 Mbps, and 15.9 Mbps respectively. From the results, it is seen that as $m$ reduces, the impact of VHOs on data rate is reduced because the rate of blocking of VLC channel is decreased as movement duration reduces. The results in Fig. 14 show that our scheme has the highest average data rate as $m$ increases beyond $3 \mathrm{~s}$ since the increasing rate of blocking has more effect on the other schemes than on CAD-VHO which minimizes the occurrence of unnecessary VHOs by adjusting the dwell time.

Finally, we investigate the effect of pause time interval $p$ of restless MT on the average data rate for the various schemes. As $p$ increases from 3 to $12 \mathrm{~s}$, the average data rate for all the schemes constantly increases. This shows the positive impact of a longer pause time interval on data rate since the rate of blocking reduces as pause time interval increases. In comparison with the I-VHO scheme, the average data rate for our scheme is increased by $13.8 \mathrm{Mbps}$ and $6.8 \mathrm{Mbps}$ when $p$ is set to $3 \mathrm{~s}$ and $12 \mathrm{~s}$ respectively. The results in Fig. 15 show that our scheme has the highest average data rate irrespective of the pause time interval by employing a dwell timer with an optimum maximum value and the ability to adjust its value according to the history of VLC accessibility.

\section{Conclusions}

In this article, we propose a dynamic D-VHO scheme that is adaptive to changes in VLC accessibility. Our objective is to avoid unnecessary handovers thereby saving $\mathrm{VHO}$ signaling cost and optimizing system performance. To achieve this, the value of our dwell timer is varied 


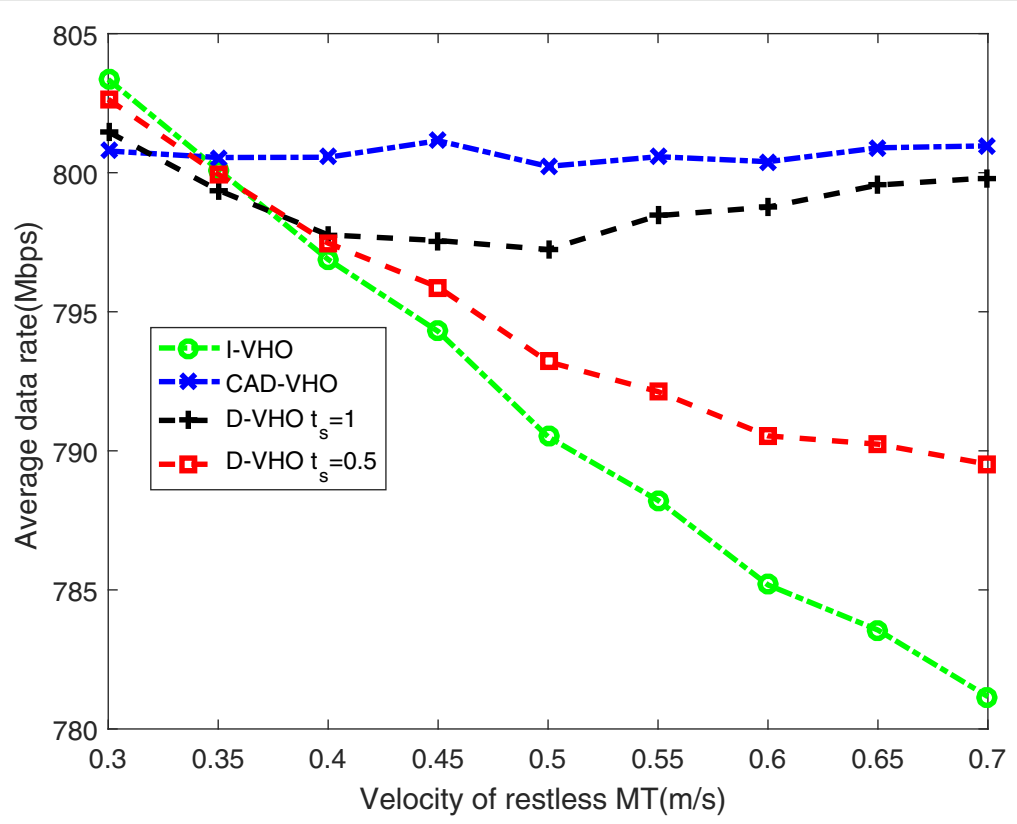

Fig. 13 Simulation results of the average data rate against velocity of restless MT

according to the rate of blocking and recovery of VLC-LoS channel. Furthermore, the proposed CAD-VHO scheme can behave like an I-VHO scheme if the rate of recovery is insignificant. The adaptive dwell timer employed is responsive to the rate and extent of physical obstruction of VLC-LoS channel and sensitive to the effect of user mobility on VLC link blocking. Simulation results show that our scheme is able to save $\mathrm{VHO}$ signaling cost by as much as $80 \%$ while achieving a higher average data rate in most instances. Therefore, the CAD-VHO scheme is highly efficient in minimizing unnecessary VHOs leading to a superior broadband service in VLC-WiFi HetNets. In future, the dwell timer adopted in our scheme can be improved to factor handover delay and other varying system conditions. Besides, the proposed channel adaptive dwell timer can be integrated into hybrid VHO schemes.

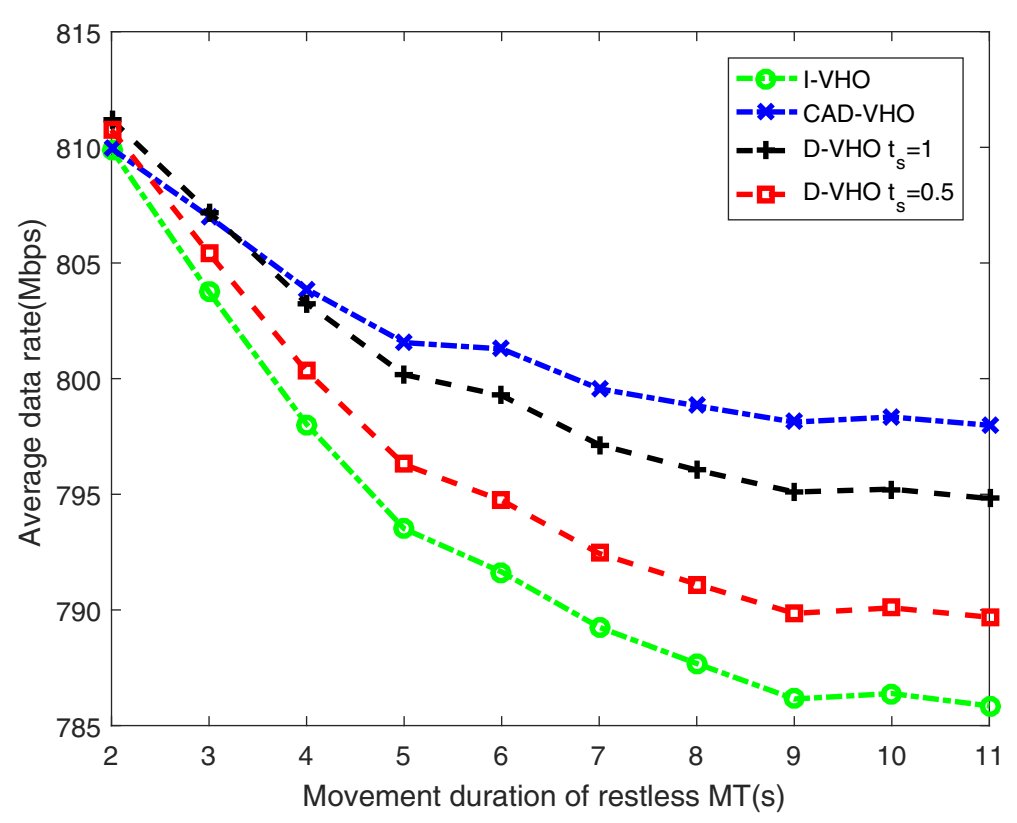

Fig. 14 Simulation results of the average data rate against movement duration of restless MT 


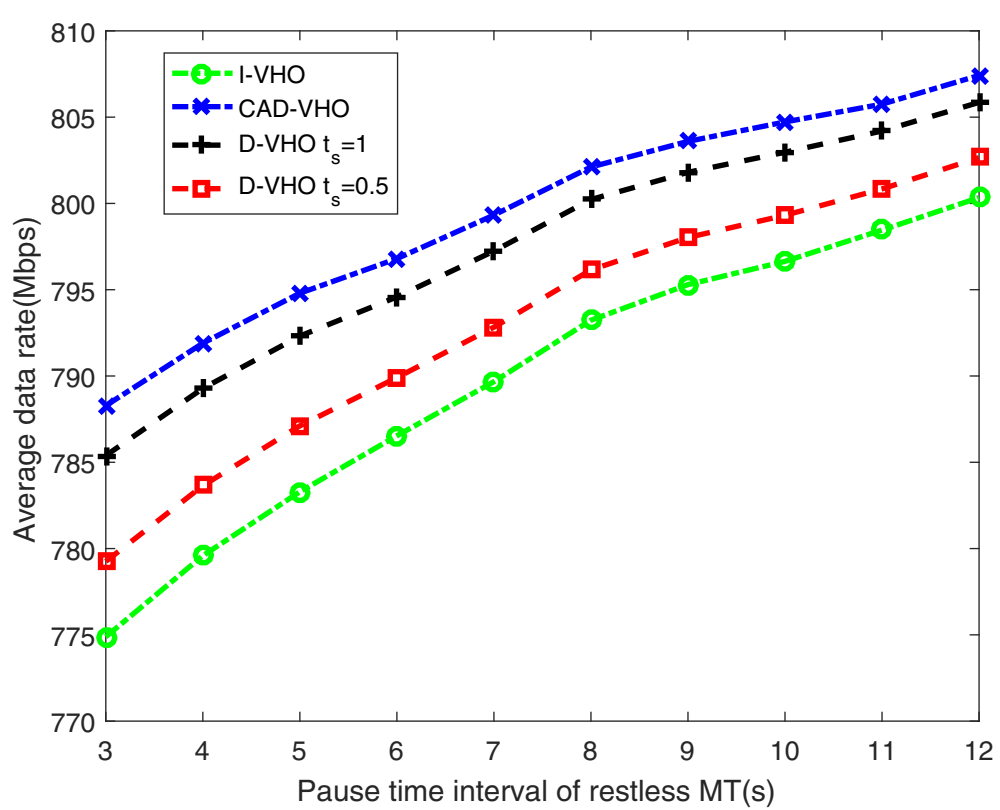

Fig. 15 Simulation results of the average data rate against pause time interval of restless MT

\section{Abbreviations}

4G: Fourth generation; AP: Access point; BS: Base station; CAD-VHO: Channel adaptive dwell vertical handover; D-VHO: Dwell vertical handover; Gbps: Gigabit per second; HD: High-definition; HetNet: Heterogeneous network; I-VHO: Immediate vertical handover; LED: Light-emitting diode; LoS: Line-of-sight; MS: Mobile station; MT: Mobile terminal; QoS: Quality of service; RF: Radio frequency; RSS: Received signal strength; TT: Time-to-trigger; VHO: Vertical handover; VLC: Visible light communication; WiMAX: Worldwide interoperability for microwave access; WLAN: Wireless local area network

\section{Funding}

This research was supported by the National Natural Science Foundation of China (Grant No. 61502210, 61772243, 61701198 and 61571211) and the China Postdoctoral Science Foundation (Grant No. 2015M570484).

\section{Availability of data and materials}

The datasets supporting the conclusions of this article are included within the article and its additional files.

\section{Authors' contributions}

XB and AA developed the CAD-VHO scheme, designed the system model, and carried out the simulation. All authors were involved in analyzing and interpreting results. $A A$ and $X B$ drafted the manuscript. WA, WZ, and JD revised the manuscript. All authors read and approved the final manuscript.

\section{Competing interests}

The authors declare that they have no competing interests.

\section{Publisher's Note}

Springer Nature remains neutral with regard to jurisdictional claims in published maps and institutional affiliations.

\section{Author details}

${ }^{1}$ School of Computer Science and Communication Engineering, Jiangsu University, 301 Xuefu Road, 212013 Zhenjiang, People's Republic of China. ${ }^{2}$ School of Electrical and Information Engineering, Jiangsu University, Zhenjiang, People's Republic of China.

Received: 8 May 2018 Accepted: 25 September 2018

Published online: 19 October 2018

\section{References}

1. M. Ayyash, H. Elgala, A. Khreishah, V. Jungnickel, T. Little, S. H. Shao, M. Rahaim, D. Schulz, J. Hilt, R. Freund, Coexistence of WiFi and LiFi toward 5G: concepts, opportunities, and challenges. IEEE Commun. Mag. 54(2), 64-71 (2016)

2. H. Burchardt, N. Serafimovski, D. Tsonev, S. Videv, H. Haas, VLC: beyond point-to-point communication. IEEE Commun. Mag. 52(7), 98-105 (2014)

3. X. Bao, X. Zhu, T. Song, Y. Ou, Protocol design and capacity analysis in hybrid network of visible light communication and OFDMA systems. IEEE Trans. Veh. Technol. 63(1), 1770-78 (2014)

4. S. Mohanty, A new architecture for $3 G$ and WLAN integration and inter-system handover management. Wirel. Netw. 12(6), $733-45$ (2006)

5. R. Zhang, J. H. Wang, Z. C. Wang, Z. Y. Xu, C. M. Zhao, L. Hanzo, Visible light communications in heterogeneous networks: paving the way for user-centric design. IEEE Wirel. Commun. 22(2), 8-16 (2015)

6. T. Nguyen, M. Z. Chowdhury, Y. M. Jang, A novel link switching scheme using pre-scanning and RSS prediction in visible light communication networks. EURASIP J. Wirel. Commun. Netw. 2013(1), 1-17 (2013)

7. X. Bao, J. S. Dai, X. R. Zhu, Visible light communications heterogeneous network (VLC-HetNet): new model and protocols for mobile scenario. Wirel. Netw. 23(1), 299-309 (2017)

8. B. V. Quang, R. V. Prasad, I. Niemegeers, A survey on handoffs - lessons for $60 \mathrm{GHz}$ based wireless systems. IEEE Commun. Surv. Tutor. 14(1), 64-86 (2012)

9. J. D. Hou, D. C. O'Brien, Vertical handover decision-making algorithm using fuzzy logic for the integrated radio-and-OW system. IEEE Trans. Wirel. Commun. 5(1), 176-185 (2006)

10. M. Ylianttila, J. Makela, K. Pahlavan, Analysis of handoff in a location-aware vertical multi-access network. Comput. Netw. 47(2), 185-201 (2005)

11. W. Lee, E. Kim, J. Kim, I. Lee, C. Lee, Movement-aware vertical handoff of WLAN and mobile WiMAX for seamless ubiquitous access. IEEE Trans. Consum. Electron. 53(4), 1268-75 (2007)

12. A. Haider, I. Gondal, J. Kamruzzaman, in 2011 IEEE 73rd Vehicular Technology Conference (VTC Spring). Dynamic dwell timer for hybrid vertical handover in $4 \mathrm{G}$ coupled networks (IEEE, Yokohama, 2011), pp. 1-5

13. X. N. Qiu, Q. Liao, in P2P, Parallel, Grid, Cloud and Internet Computing (3PGCIC), 2014 Ninth International Conference on. A vertical handoff decision algorithm based on the movement-aware of mobile nodes (IEEE, Guangzhou, 2014), pp. 54-61 
14. J. Madaan, I. Kashyap, Vertical handoff decision algorithm based on PRSS and dwell time. In. J. Comput. Netw. Inf. Secur. 8(7), 31-42 (2016)

15. Y. Li, M. Li, B. Cao, Y. Wang, W. J. Liu, Dynamic optimization of handover parameters adjustment for conflict avoidance in long term evolution. China Commun. 10(1), 56-71 (2013)

16. B. Sridevi, K. Karthikeyan, T. Meenavishakan, P. Reminbabu, S. Rajaran, Implementation of dwell timer for fast WiMAX handoff of ping pong calls. Procedia Eng. 38, 3127-38 (2012)

17. I. Smaoui, F. Zarai, M. M. Banat, L. Kamoun, Heterogeneous wireless networks: configuration and vertical handoff management. Wirel. Pers. Commun. 54(3), 417-45 (2010)

18. B. Liang, A. H. Zahran, A. O. M. Saleh, in 2005 4th International IFIP-TC6 Networking Conference (NETWORKING 2005). Application signal threshold adaptation for vertical handoff in heterogeneous wireless networks (Springer, Waterloo, 2005), pp. 1193-1205

19. F. Wang, Z. C. Wang, C. Qian, L. L. Dai, Z. X. Yang, Efficient vertical handover scheme for heterogeneous VLC-RF systems. IEEE/OSA J. Opt. Commun. Netw. 7(12), 1172-80 (2015)

20. R. Liu, C. Zhang, in 201713 th International Wireless Communications and Mobile Computing Conference (IWCMC). Dynamic dwell timer for vertical handover in VLC-WLAN heterogeneous networks (IEEE, Valencia, 2017), pp. 1256-60

21. S. F. Liang, H. Tian, B. Fan, R. L. Bai, in 2015 IEEE 82nd Vehicular Technology Conference (VTC Fall). A novel vertical handover algorithm in a hybrid visible light communication and LTE system (IEEE, Boston, 2015), pp. 1-5

22. C. Yan, Y. Xu, J. Shen, J. Chen, in 2016 IEEE International Conference on Ubiquitous Wireless Broadband (ICUWB). A combination of VLC and WiFi based indoor wireless access network and its handover strategy (IEEE, Nanjing, 2016), pp. 1-4

23. Y. Wang, X. Wu, H. Haas, in 2016 IEEE International Conference on Communications (ICC). Fuzzy logic based dynamic handover scheme for indoor Li-Fi and RF hybrid network (IEEE, Kuala Lumpur, 2016), pp. 1-6

24. M. S. Saud, M. Katz, H. Haas, in 201723 th European Wireless Conference. Implementation of a hybrid optical-RF wireless network with fast network handover (VDE, Dresden, 2017), pp. 1-6

25. S. Liang, Y. Zhang, B. Fan, H. Tian, Multi-attribute vertical handover decision-making algorithm in a hybrid VLC-femto system. IEEE Commun. Lett. 21(7), 1521-24 (2017)

26. X. Bao, G. D. Yu, J. S. Dai, X. R. Zhu, Li-Fi: light fidelity-a survey. Wirel. Netw. $\mathbf{2 1}(6), 1879-89(2015)$

27. J. H. Liu, Q. Li, XY. Zhang, Cellular coverage optimization for indoor visible light communication and illumination networks. J. Commun. 9(11) (2014)

28. N. Chi, J. Shi, Investigation on overlapping interference on VLC networks consisting of multiple LEDs. ICT Express. 1(2), 63-66 (2015)

29. H. Liu, J. Zhang, Sci. China Ser. F Inf. Sci. 51(8), 1154-1166 (2008)

\section{Submit your manuscript to a SpringerOpen ${ }^{\circ}$ journal and benefit from:}

- Convenient online submission

- Rigorous peer review

- Open access: articles freely available online

- High visibility within the field

- Retaining the copyright to your article

Submit your next manuscript at $\gg$ springeropen.com 